\title{
NON-LINEAR 3D FINITE ELEMENT ANALYSIS OF THE FEMUR BONE
}

\author{
Sivakumar V $\mathbf{1}^{\mathbf{1}}$, Asish Ramesh U. $\mathbf{N}^{\mathbf{2}}$ \\ ${ }^{1}$ Associate Professor, Department of Aerospace Engineering, ${ }^{2}$ Post Graduate Student, Department of Mechanical \\ Engineering, Amrita Vishwa Vidyapeetham University, Coimbatore, Tamil Nadu, India, v_sivakumar@cb.amrita.edu
}

\begin{abstract}
In this paper a 3D stress analysis on the human femur is carried out with a view of understanding the stress and strain distributions coming into picture during normal day to day activities of a normal human being. This work was based on the third generation standard femur CAD model being provided by Rizzoli Orthopedic Institute. By locating salient geometric features on the CAD model with the VHP (Visible Human Project) femur model, material properties at four crucial locations were calculated and assigned to the current model and carried out a nonlinear analysis using a general purpose finite element software ABAQUS. Simulation of Marten's study revealed that the highest stress formed in the absence of the cancellous tissue is almost double the value of stress formed with cancellous tissue. A comparative study was made with the Lotz's model by taking into consideration two different sections near the head and neck of the femur. An exhaustive number of finite element analyses were carried out on the femur model, to simulate the actual scenario.
\end{abstract}

Index Terms: Fracture, Cortex, Cancellous, femur bone, finite element

\section{INTRODUCTION}

Femur bone or the thigh bone is the largest and strongest bone in the human body, refer figure 1. It acts as one of the most prominent load carrying member apart from the spinal column. So it is only natural that any sort of impairment could seriously affect the day to day activities of the concerned individual. Medical complications such as osteoporosis, arthritis etc, can increase the chances of a hip fracture in cases of accidents. The term hip fracture is actually a misnomer; as it refers to the fracture occurring in the femur bone itself. One of the operative procedure on fractured femur bone (Fig. 1) containing a crack which can be internally fixed by means of holding screws. Here, a screw is driven through the thickest part of the bone such that, once, tightened, the screw clamps the broken part of the bone to the existing part of the bone, and in due course of time leads to the healing of the bone at the cracked region. The function of the screw in such cases are multipurpose; viz; holding the cracked region of the bone on to the existing part, such that natural healing occurs and also to bear the individuals weight till the bone attains its actual strength.

In order to properly simulate the loading condition of the bone, knowledge of the biomechanics involved is required. There are three loading conditions usually considered in finite element (FE) analysis of the femur. These loading conditions are similar to the anatomical loading in the actual femur. They are: Static one-legged stance, Gait loading, Impact loading, or loading during fall. Of these, static one legged stance was taken up in the present study and is the simplest of the above three. Here, the individual is assumed to be standing on one leg. When the weight of the body is being borne on both legs, the center of gravity is centered between the two hips and its force is exerted equally on both hips. When the individual shifts to one leg, the centre of gravity of the body shifts away from the supporting leg and a large force will have to be sustained by the supporting leg.

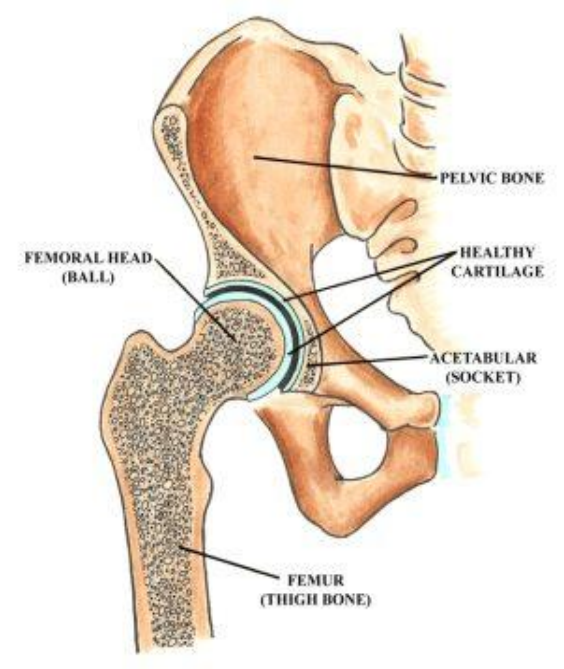

Fig 1: The human hip joint

Large muscle forces come into the picture to counteract the newly created moment. There are approximately nineteen muscles that come into action in the hip region and an inclusion of all these muscles in the analysis is virtually impossible. Hence, for the sake of simplicity, vertical load which is representative of the resultant forces are considered in this analysis. 
The bone consists of an outer dense bone called cortex and an inner bone region called cancellous tissue which is arranged in a lamellar fashion and is continuous with the cortical region. The cortex is densely packed and the cancellous tissue is arranged in the form of crisscrossing lamellae. Bone tissues, whose percentage of solid matter are less than $70 \%$ in a given volume can be classified as cancellous. A cut away section of the femur bone is shown in figure 2 for clarity. The different areas of the femur are classified into: Epiphysis, Metaphysis, Diaphysis. Epiphysis is the bone area corresponding to the head of the femur. Diaphysis is the area corresponding to the shaft of the femur; and metaphysis is the area of the bone that connects the above two. The density of the cortical tissue is very less in the epiphysis region and as it extends down the shaft, the thickness of the cortical tissue gradually increases.

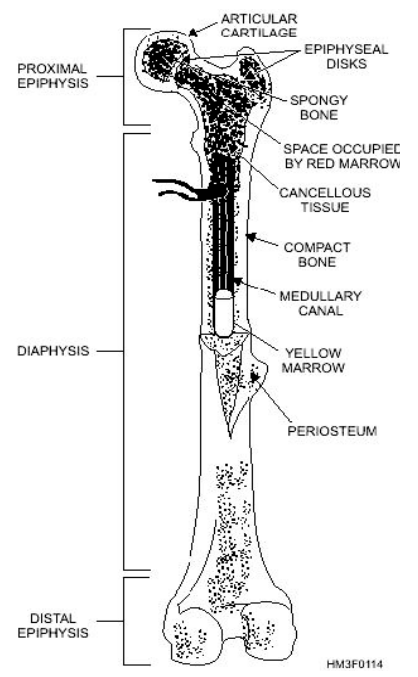

Fig-2: The human hip joint

Thus, the middle diaphysis region has the densest cortical tissue and the proximal and distal ends have the densest cancellous tissue. The diaphysal region has a thin hollow region called medullary canal, inside which is present the soft yellow and red marrow tissues. The proximal part of the femur bone was investigated in this project as it is of a complex geometry and almost all points of loading fall in this area. The proximal part of the femur is shown in figure 3 .

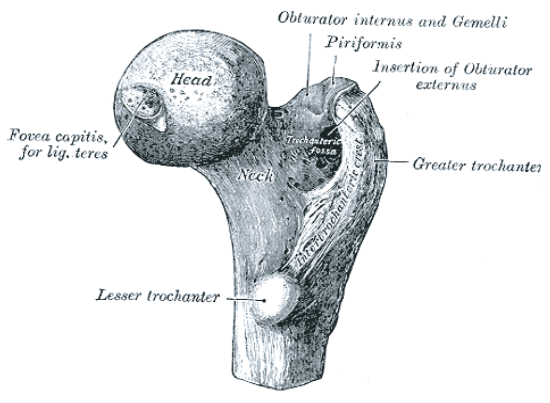

Fig-3: The proximal femur

\section{LITERATURE REVIEW}

As long as in the early 1970s itself, finite element analysis has been an accepted method for analyzing the structural capacity of bovine and human femur bones. Researchers made great strides in designing better prosthetics due to studying internal stress and shear behavior of FE models. For example, Huiskes et al. [1], investigated stem flexibility based on strain adaptive bone remodeling theory. This study was virtually impossible without using finite elements since there exists no other method capable of determining internal behavior. Conclusions from his study showed flexible stems reduced stress shielding and bone resorption, however, increasing proximal interface stresses. Hampton et al. [2] verified stress distributions responsible for most clinically observed failures in hip prosthesis. In addition, Brown et al. [3] investigated stress redistribution in femoral head from bone osteonecrosis. FEA continues to be useful since it can replicate osteoporotic conditions in a FE model and have the ability to iterate many types of load cases in one study. Frequently, experimental studies are compared to FE models for validation. Brown et al. [4] studied the Stress redistribution in the adult hip resulting from property variations in the articular regions of the femoral head. In McNamara [5], the experimental and numerical strains of an implanted prosthesis is compared. Stress analysis of femoral stem of a total hip prosthesis was done by Hamptton et al. [2]. Huiskes et al. [1] studied the relationship between stress shielding and bone resorption around hip stem. Little et al. [6] were worked on proximal tibia stress strain prediction. Lotz et al. [7] carried out a linear analysis of proximal femur fracture and Rohlmann et al. [8] investigated the stresses in an intact femur.

\section{FINITE ELEMENT MODELING}

In order to carry out FEA of any components, the first and the basic requirement is a $\mathrm{CAD}$ model; either $2 \mathrm{D}$ or $3 \mathrm{D}$, depending on the user requirements. Usually, a CAD model of a complex geometry in biological systems as in case of the femur bone is obtained from computerized tomographic scans (CT). The CT scan data is first obtained in a special format called DICOM format, which provides a lot of information about the cross sections of the bone, in vivo and in vitro. Using this information, one can obtain the CAD model of that particular scanned bone using commercially available software like 3D doctor. Additionally, these scans measure the bone density as well, which can be correlated to the strength properties of the bone. The CAD model of this surrogate was modeled by Rizzoli Orthopedic Institute from the CT scan of the third generation composite model, which has been made freely available over the internet for academicians. Thus, the third generation composite femur has become the common platform on which all research works are based, so that the results become comparable. The current work is based on the third generation composite femur. The third generation composite femur, which is freely downloadable from the Rizzoli Orthopedic Institute website, was downloaded in Pro- 
Engineer format. The standardized femur was first truncated to a length of $150 \mathrm{~mm}$ from the top of the head along the shaft in Pro-Engineer. This was done so as to reduce the number of elements coming into picture while meshing and also to reduce computational efforts. The truncated model of the femur is shown in figure 4. Also, previous convergence studies have proved that the variation in von Mises stress near the proximal region of the femur ceases once past $150 \mathrm{~mm}$ along the shaft [9].

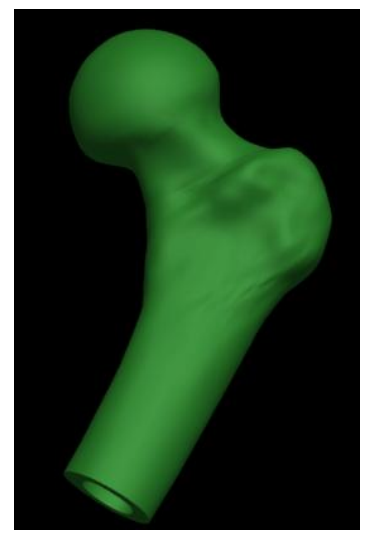

Fig-4: The truncated model of the 3rd generation composite femur

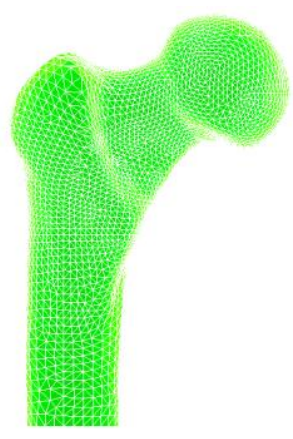

Fig-5: 3D meshed model of Femur

The truncated model obtained in Pro-Engineer format was imported to Hypermesh. Since Abaqus was the intended solver, the particular template was activated. Some geometrical inconsistencies present in the CAD model were fixed up using Hypermesh. The model actually consisted of two surfaces; one representing the outer cortex region and the next representing the inner cancellous region. Two new surfaces were additionally required to cover up the base that was now left open due to the truncation operation that was done in Pro-Engineer. Meshing of the two volumes were carried out separately. The meshing for the stronger cortical was made finer and dense mesh was applied to the complicated contours in the proximal area. The two meshed volumes corresponding to the cortical tissue and the cancellous tissue was then combined together to obtain the complete meshed model as shown in figure 5. The Material properties corresponding to these meshed regions were defined. In the present study, a contact was defined at the interface between the cortical and cancellous tissue. This approach is different from the previous works wherein the two types of tissues namely cortical and cancellous used to be one single meshed model, with the material properties for each of them defined separately. Since contact analysis came into picture, the problem became a non-linear one. Many different analyses were carried out to have a proper understanding of the stresses and strains in the intact femur. They are discussed in the following sections.

\subsection{Material Properties of the Bone}

It is important that the material properties of the cortical and cancellous tissues be understood before one attempts to carry out any analyses on them. Cortical bone is made up of a solid external layer throughout the walls of the diaphysis and the external surfaces of bone. Reilly and Burstein [15] conducted exhaustive studies on the cortical tissue and concluded that modulus values were similar in both tensile and compression tests. All FEA studies carried out in this project is based on the assumption that cortical and cancellous bones mechanically behave linearly before yield. CT scans have become popular in defining material properties because it can associate material property values to a FE model. Researchers like Lotz et al. [9], Keyak et al. [11], and Carter and Hayes et al. [12] used the CT method to associate material properties to their FE models. There are several techniques employed for determining material properties of cortical bones refer [7, 10, 13-16]. Many authors have shown cortical bone to contain a homogeneous distribution throughout the cortical region, but few authors such as Lotz [7] modeled cortical bone moduli dependent on the thickness of the bone. Lotz [9] in his experimental setup determined the three categories of cortical bone as diaphysis, metaphyseal, and reduced thickness. The reduced sections were defined as bone with a $1 \mathrm{~mm}$ thickness or less. Moduli for each of the three classifications of bone were determined using the load-deflection data from 3-point bending tests. Shear modulus components were obtained from Reilly and Burstein [15]. All values are shown in Table 1.

Table-1: Material properties for the cortical tissue

\begin{tabular}{|c|c|c|c|c|c|c|c|c|c|}
\hline \multicolumn{8}{|c|}{ Cortical material properties for linear model } \\
\hline Location & $\begin{array}{c}\mathbf{E}_{\mathbf{1}} \\
\mathbf{M P a}\end{array}$ & $\mathbf{E}_{\mathbf{2}}$ & $\mathbf{E}_{\mathbf{3}}$ & $\mathbf{E}_{\mathbf{1 2}}$ & $\mathbf{E}_{\mathbf{2 3}}$ & $\mathbf{E}_{\mathbf{3 1}}$ & $\mathbf{v}_{\mathbf{1 2}}$ & $\mathbf{v}_{\mathbf{1 3}}$ & $\mathbf{v}_{\mathbf{2 3}}$ \\
\hline Diaphysis & 11 & 11 & 16.3 & 3.46 & 3.15 & 3.15 & .58 & .31 & .31 \\
\hline Metaphyseal & 7.4 & 7.4 & 11 & 2.31 & 2.11 & 2.11 & .58 & .31 & .31 \\
\hline Reduced & 2.8 & 2.8 & 3.5 & .9 & .82 & .82 & .58 & .31 & .31 \\
\hline
\end{tabular}


It was felt that Lotz's model could be representative of the actual bone structure as it represented an orthotropic model and also the variation in strength with change in location is also taken into account. Hence, in this project, these properties are considered.

Cancellous bone, also known as trabecular bone, is located in the epiphyseal and metaphyseal region of long bones and has 30 percent greater porosity than cortical bone [17]. Cancellous bone plays an extremely important role in mechanical behavior of femur bones. In a study involving the total absence of the cancellous bone in the head and neck region of the femur, it was found that the cancellous bone accounts for approximately half the femur strength [18]. This has been verified in this study. References [17-24] show the variation of cancellous bone material properties derived from various test methods such as tensile, compression, and ultrasonic. It can be seen from the literature, unlike the values obtained for cortical bone's material properties, those obtained for cancellous showed a large variation among them. Moduli values depend on direction of testing while both modulus and ultimate strength are dependent on health of bone.

\section{RESULTS AND DISCUSSIONS}

The results of 3D FE analyses were broadly divided into four sections. In first section, Marten's study on femur bone was carried out to validate the current FE model. In the second section, the realistic model was formed with variation of densities within the cancellous region. In the third section the present model is compared with the Lotz's model and in the fourth section the analysis was carried out on the Intertrochanteric region.

\subsection{Verification of Marten's Study}

Martens [17], in one of his studies came up with an interesting observation that, in the complete absence of cancellous tissue in the bone, the strength or the load carrying capacity of the bone reduces by $50 \%$. An analysis was carried out to verify the same. Initially, the whole of the cancellous tissue was avoided to obtain the bone composed only of the outer cortical tissue. As discussed previously, bone is more of an orthotropic nature than isotropic, and Lotz [9] in his one of his studies assigned material properties depending on the thickness of the cortical tissue. To have a realistic analysis, the cortical tissue was assumed as orthotropic as in Lotz's study. The Young's modulus values were taken from Lotz's data, and the shear modulus components were obtained from Reilly and Burstein [15]. The material properties are already shown in table 1 . It proved difficult to calculate the thickness at proximal region, though the thickness of the cortical tissue in the diaphysal region could clearly be visualized. So, the whole of the cortical tissue was approximated as having properties corresponding to the metaphyseal region. Also, it can be seen from the table that material properties at the metaphyseal region is almost equal to the average values at reduced and diaphyseal regions. The specifications were as follows: Vertical load of $450 \mathrm{~N}$ was applied over nine nodes at the top of the femoral head by arresting all degrees of freedom at the base, Static non-linear analysis was carried out in Abaqus.

For estimating the contribution of cancellous tissue towards the strength of the bone, the von Mises stress distribution that developed was considered. Figure 6 shows the von Mises stress distribution for the bone without cancellous tissue. From

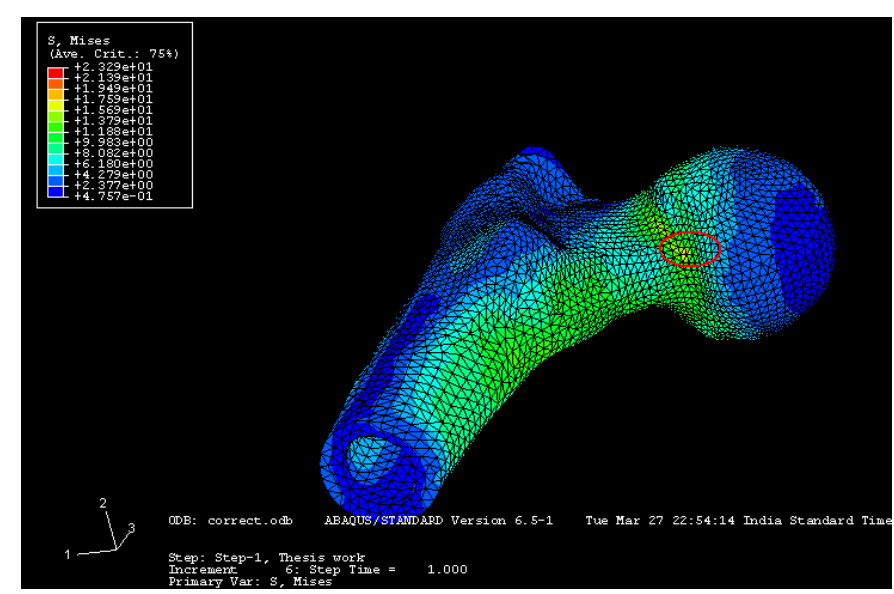

Fig-6: von-Mises stress distribution for the bone without cancellous tissue

the figure, it can be seen that the maximum stress developed is 23.29MPa. The location of this stress is shown circled in the figure. Figure 7 shows the same bone with the same loading conditions with the cancellous tissue included. Cancellous tissue in this case was defined to be having isotropic properties with a Young's modulus of $1000 \mathrm{MPa}$ and a Poisson's ratio of 0.3 [12].

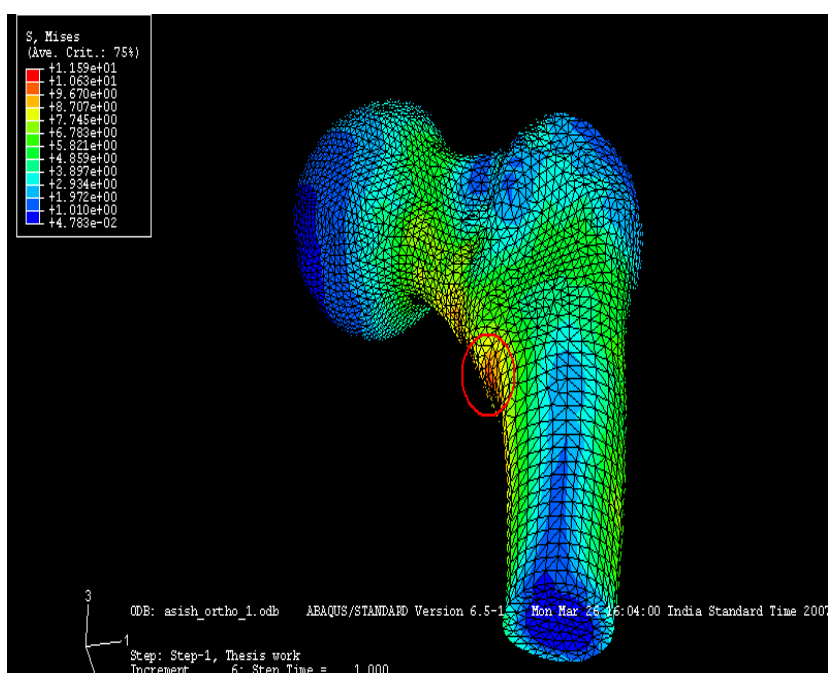

Fig-7: von-Mises stress distribution for the bone with cancellous tissue 
In comparison, the stress highest stress developed in the second model, that is, the model in which the cancellous tissue was included was found to be much lower with a value of 11.59MPa. The location of this stress is indicated with a red circle. In the model without the cancellous tissue, the reason for the maximum stress originating at the immediate base of the head (see Fig. 6) could be attributed to the geometrical properties of the model. Near the head region, the thickness of the cortical tissue is very less. This, along with the fact that the region below the head would be the most stressed in a loading condition as this could have contributed towards the occurrence of this phenomenon. Considering the bone with the cancellous tissue, the highest stress is found to be occurring at a lower region (see Fig. 7). This indicates that the presence of cancellous tissue has strengthened the bone and the most stressed out region has now shifted to the new location which could be a vulnerable one in terms of strength bearing. The nodal deformations of these cancellous and non-cancellous tissue models are shown in figure 8 and 9 respectively. A highest value of $0.91 \mathrm{~mm}$ deflection was observed in case of the bone with the cancellous tissue and a highest value of $1.147 \mathrm{~mm}$ deflection was observed in case of the bone without cancellous tissue. An increase of $21 \%$ was observed in the deflection values in the absence of the cancellous tissue.

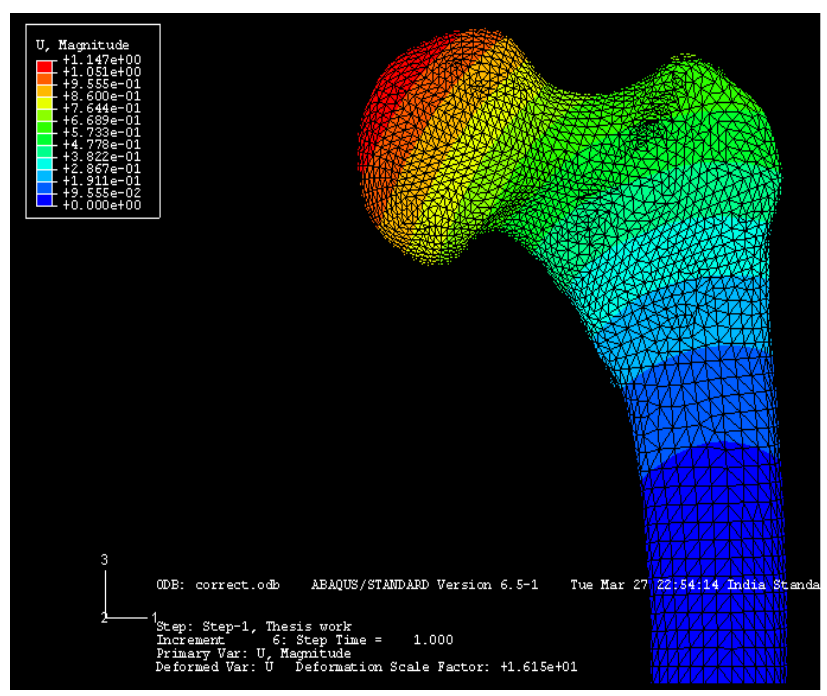

Fig-8: Nodal displacements for the model without cancellous tissue

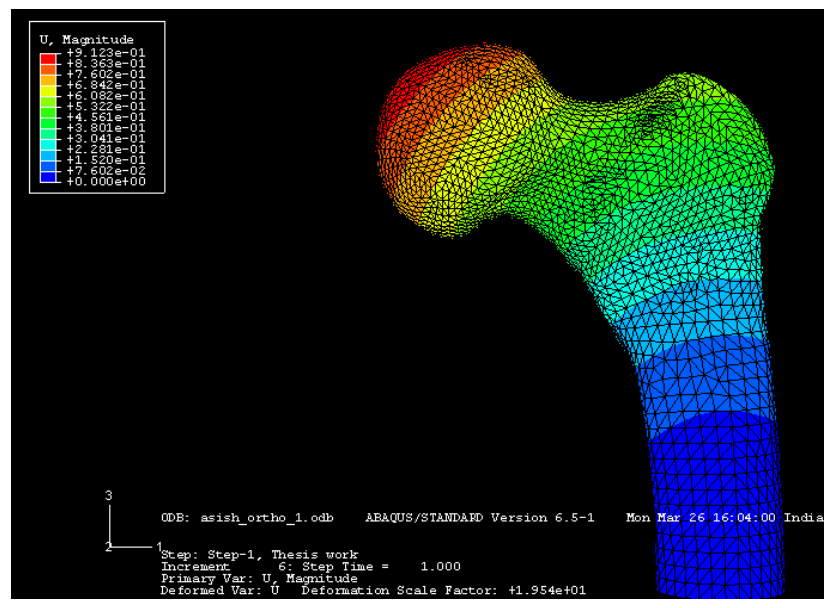

Fig-9: Nodal displacements for the model with the cancellous tissue

\subsection{Formation of a Realistic FE Model}

In the second study, an attempt was made to bring the model closer to the actual scenario by considering the variation in density within the cancellous tissue. The usual techniques of obtaining the CAD model are incapable of providing any information regarding the density distribution of the bone. However, CT scans are capable of providing density distribution of the bone in addition to defining the complicated contours properly. The density distribution thus obtained can be used to correlate and arrive at the material property of the bone at that particular location. The fifteen sections that Eric [24] used in his paper was taken up and further reduced to four sections. Thus, in the new model, the cancellous tissue was further divided into four sections having isotropic properties. The modulus values for each of these sections were calculated by averaging the modulus values present at the end sections. The cancellous tissue which was subdivided into four regions is as shown in figure 10. The moduli value decreased as one came down the bone from the top of the head. This cancellous material was then combined with the cortical tissue to obtain the complete femur model. Static non-linear analysis was carried out with the same conditions. 


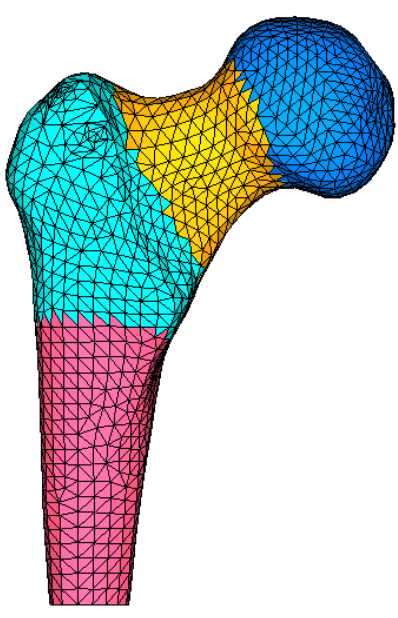

Fig-10: Sub-divisions made in the cancellous tissue

The stress distribution along the vertical axis of the femur is shown is figure 11. It can be seen from the figure that the extreme compressive and tensile values are felt at the inner and outer regions of the bone. The region where the diaphysis ends experiences high compressive stress and the area at the outer extreme of the diaphyseal region experiences tensile stress. The areas with highest tension and compression values are found to be at the inside and outside sides of the diaphyseal region where all degrees of freedom were arrested. The highest compressive and tensile stresses at the base are $22.81 \mathrm{MPa}$ and 19.34MPa respectively which are almost of the same magnitude. It has to be noted that these high values

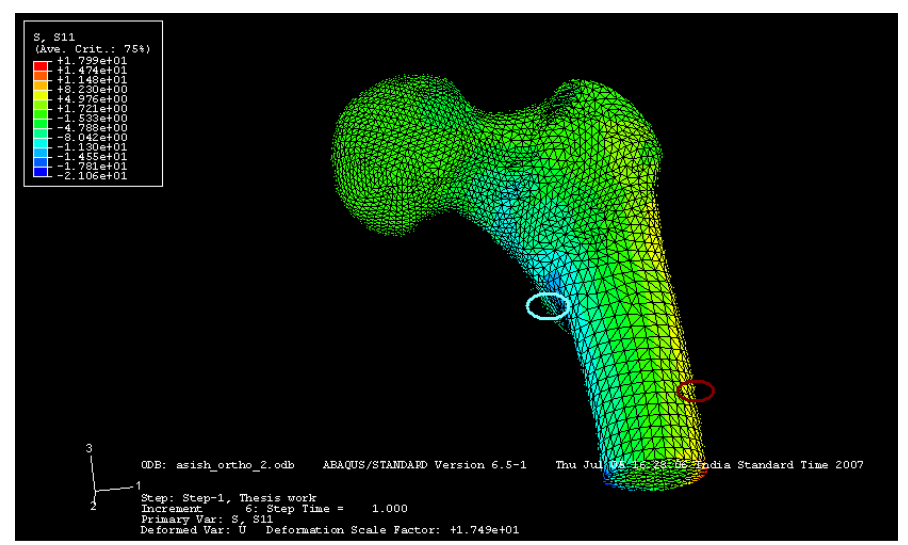

Fig-11: Stress distribution for cortical tissue along the diaphyseal axis

could be a result of arresting all degrees of freedom at the base. However, at the diaphyseal region these values are -21.4 $\mathrm{MPa}$ ( circled in cyan) and $12.56 \mathrm{MPa}$ (circled in red) respectively. The difference in the magnitude of these values can be attributed to the fact that tensile stresses are distributed over a larger area when compared to the area upon which the compressive stresses act. Figure 12 show the stress variation in the cancellous tissue.

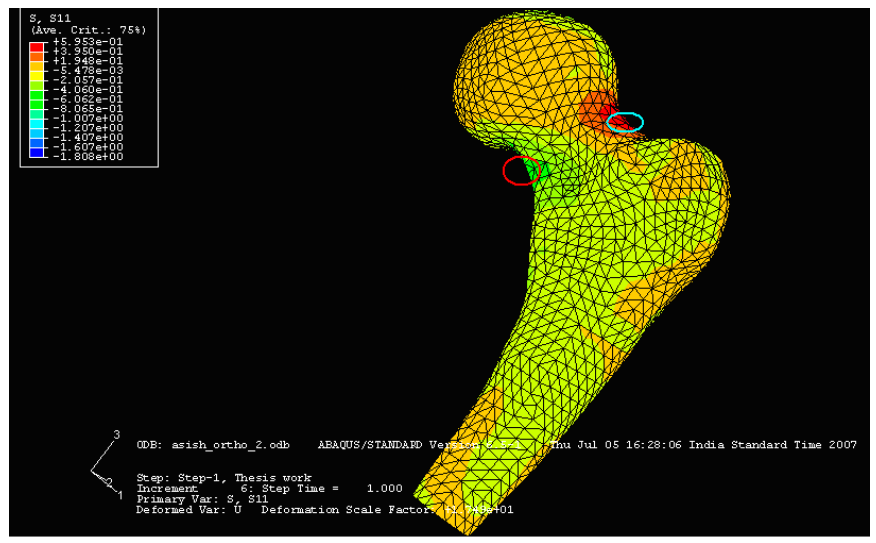

Fig-12: Stress distribution in the cancellous tissue along the diaphyseal axis

The cancellous tissue is also found to exhibit almost the same type of stress distribution as in the case of cortical tissue, the difference being that some material at the outside fibers was under compressive stress and not tensile as in the case of cortical tissue. This could be attributed to the combined effect of contact specified at the cortical cancellous transition region. However, at the neck region, compressive stresses were felt at the base of the neck, registering a highest value of $-0.961 \mathrm{MPa}$ (indicated in red circle) and the top of the neck experienced the highest tensile stress, registering a value of $0.744 \mathrm{MPa}$ (indicated in cyan circle). This behavior was in perfect agreement with that expected.

\subsection{Comparison of Present study with Lotz's Study}

Lotz [7] in his study had considered 15 sections starting from the top of the femoral head, and extending into the diaphyseal region. For sake of validation, two sections, namely section 5 (indicated in green colour) and section 7 (indicated in red colour) were considered in the present study model in figure 13. Section 7 was considered because, this was found to be a high stress region, and section 5 was chosen so as to understand how the stress varied from a region of high stress to a lower one. In this model, apart from using orthotropic properties for the cortical tissue, subdivision of cancellous tissue into four regions having different isotropic properties using the CT scan data, an adduction angle of 11 degrees with the horizontal corresponding to one-legged stance has also been taken into account [9]. Adduction angle is the clock-wise angle by which the vertical axis passing through the axis of the diaphyseal region has rotated. Adduction of the femur is the body's natural response to reduce the stress induced when an individual shifts to one leg. This model thus represents the best approximation of the actual scenario, in the sense that material, as well as geometrical properties was taken into account. Figure 13 shows the femur adducted at an angle of 11 degrees and the sections 5 and 7 which were considered for comparison purpose. Please note that only the cancellous tissue is shown. The cross-sectional views of section 5 and 
section 7 with the von Mises stress distribution is shown in figure 14 and 15.

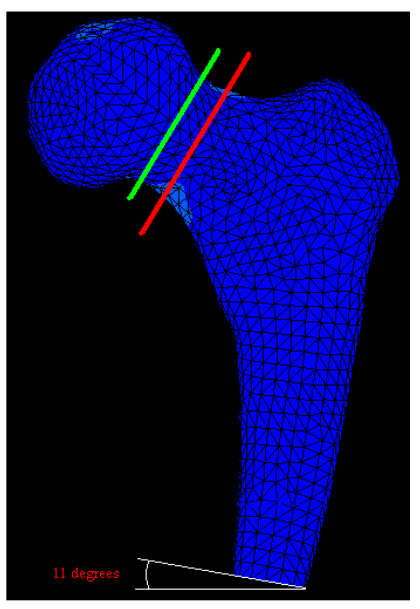

Fig-13: Section 5 (indicated in green) and section 7 (indicated in red)

From the figure it can be seen that the highest and lowest values for both the sections occur at the anterior and posterior sides. For section number 7 , the highest stress recorded was 9.01 to $9.35 \mathrm{MPa}$ (indicated by the purple circle at the interior side, the red circle indicates the area of lowest stress). For the Lotz's model, these values were respectively $7.5 \mathrm{MPa}$ and $9 \mathrm{MPa}$. For section number 5, the highest stress values recorded was 6.7 to $6.9 \mathrm{MPa}$ (indicated by the red circle in the

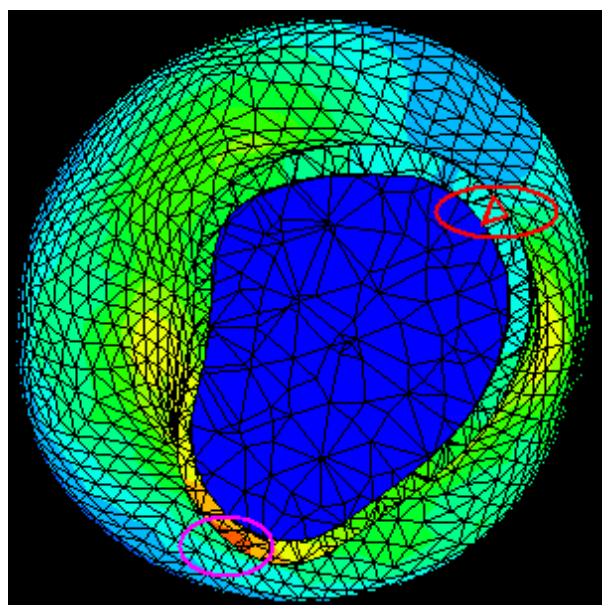

Fig-14: Cross-sectional view of section 7

anterior area, the green circle indicates the area of the lowest stress). For the Lotz's model, these values were $4 \mathrm{MPa}$ and 4.28MPa respectively. The stress distribution for the Lotz's model for section number 7 and 5 is shown in figure 16. It can

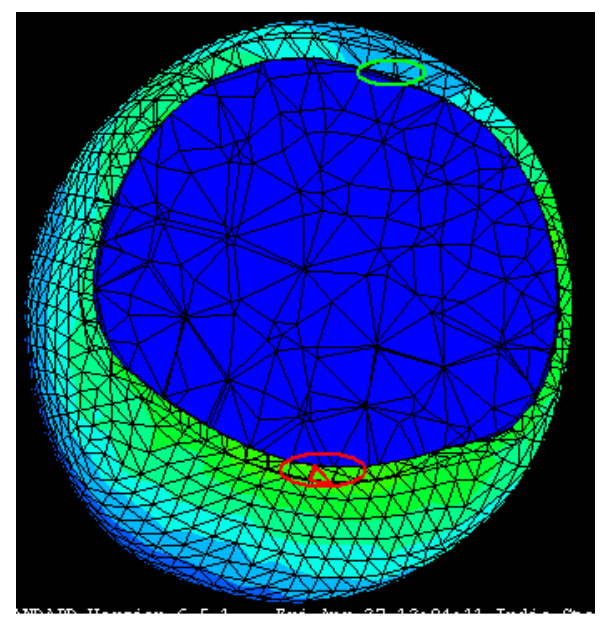

Fig-15: Cross-sectional view of section 5

be observed that the location of the stresses is almost similar in the current model as well as Lotz's model. It can be seen that the current model is very well capable of predicting the high stress formation in the neck region, though the low stress values obtained at the neck region for the current model varied

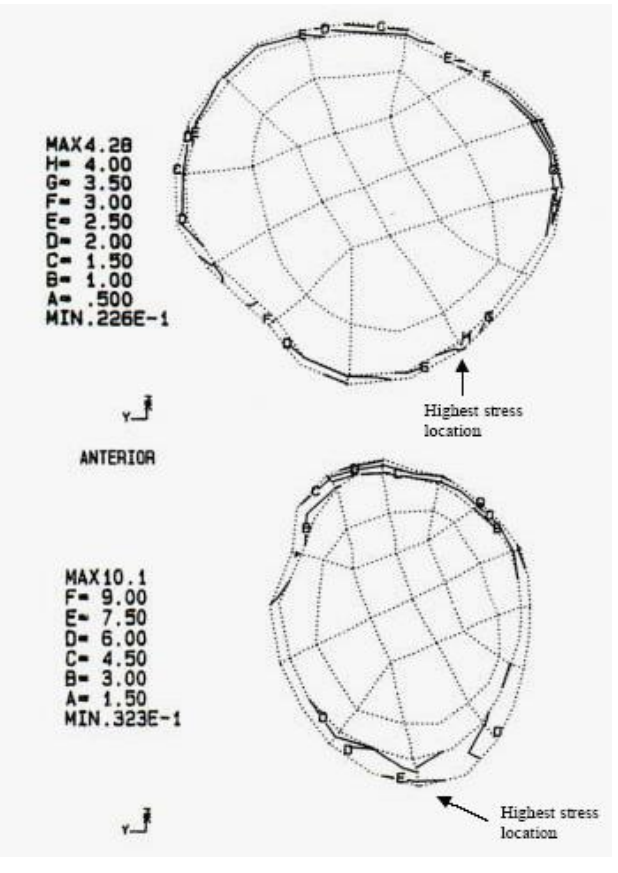

Fig-16: Stress distribution for the Lotz's model at sections 5 and 7

Significantly from that obtained in Lotz's study [7]. The low stress value at the beginning of the head region in the current model varied by $61 \%$ when compared to the low stress value obtained in Lotz's model. Figure 17 shows the stress gradient along section 7 . 


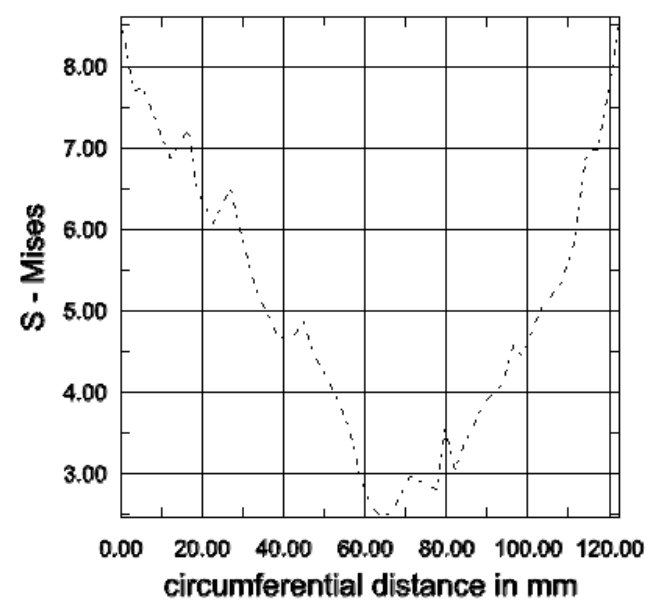

Fig-17: Circumferential variation of von Mises stress at section 7

From the graph it can be seen that the stress felt is at its highest at the anterior region, and as it proceeds along the loop, it reduces to its minimum at the posterior region and again starts increasing towards the anterior side. It is interesting to note that the lowest stress is felt almost at the half of the loop length, i.e.; at $60 \mathrm{~mm}$, which is indicative of a symmetry in stress distribution.

\subsection{Analysis of the Intertrochanteric Region}

If the intertrochanteric bone subjected to a crack then deals with the fixation devices used at the intertrochanteric region. Hence an investigation at the intertrochanteric region was felt necessary. Figure 18 shows the axial stress distribution along the diaphyseal axis for the above discussed bone model. The

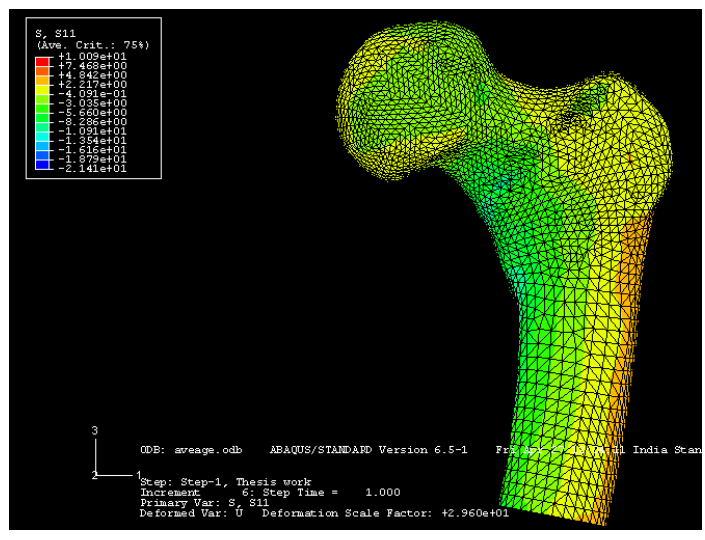

Fig-18: Axial stress distribution for the bone

Adduction angle of 11 degrees was considered as this had brought the results closer to the values in literature. The model used here is the same as in section 4.3.
It was found that the maximum tensile stress was found in the posterior diaphyseal region and the maximum compressive stress in the anterior diaphyseal region (at the beginning of the diaphyseal region). However, there are many reported cases of intertrochanteric failures in accidents involving the hip. This could be attributed to the fact that intertrochanteric region is a transition region from the metaphyseal region to the diaphyseal region and a reduction in strength could be inherent to this region. However, it was decided to investigate the intertrochanteric area for its behaviour during loading of the femur. A section immediately below the intertrochanteric line was selected for the purpose. For understanding the stress gradient along the intertrochanteric plane, the stress distribution along the outer periphery of the cortical bone was considered and the respective stress gradation is shown in figure 19. From the graph it can be seen that, unlike the stress distribution at the neck region, it is not exactly symmetrical. The highest compressive stress is at a circumferential distance of about $75 \mathrm{~mm}$ from the point where highest tensile stress was present and has a magnitude of about $-10 \mathrm{MPa}$. The tensile stress peaks the graph at around $2.5 \mathrm{MPa}$. Another point that can be observed from the graph is that the magnitudes of compressive stresses are higher in this region, though their distribution is more or less similar.

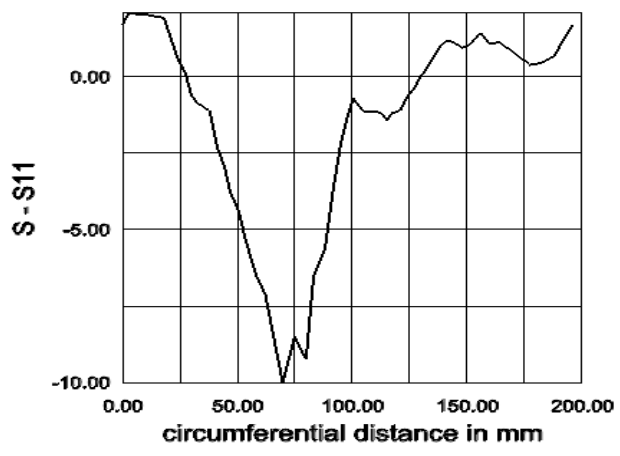

Fig-19: Circumferential stress variation at the intertrochanteric

Comparing to the stress distribution at section 7 discussed previously, the stresses are distributed in a symmetrical manner as shown in figure 20. It can be seen that in case of stress gradation at section 7 also, the predominant stresses are compressive in nature. A plot depicting the magnitude of displacement for different nodes along the z-direction at the intertrochanteric region is shown in figure 21 . To interpret the relationship of nodal displacements with varying stress values, a plot of nodal displacements verses stresses were plotted as shown in figure 22. It was found that the trend line for the 


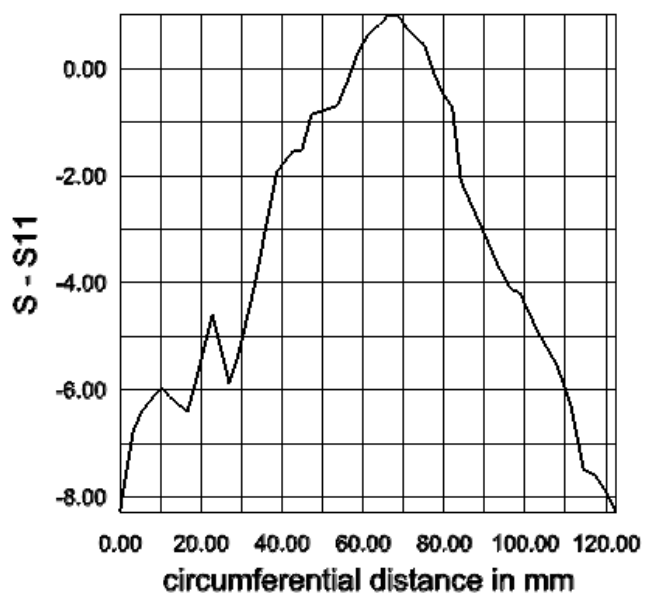

Fig-20: Circumferential stress variation at section 7

different data points can be approximated as linear for these values (shown by the straight line in the figure). This implies that the stress-displacement relationship at the intertrochanteric region could be considered as linear. In other words, stress developed was found to be directly proportional to strain.

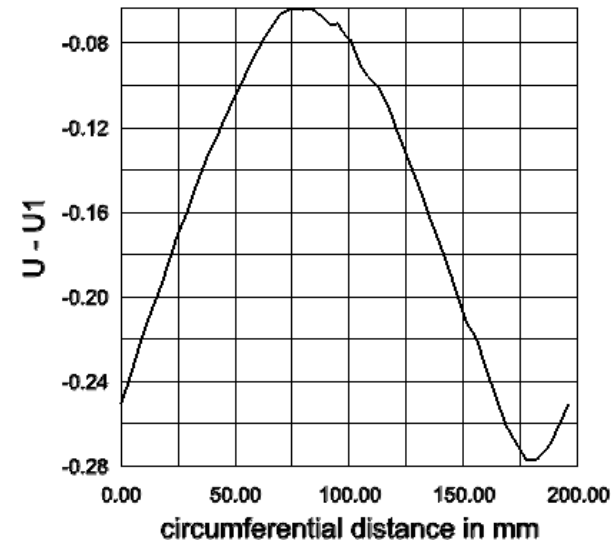

Fig-21: Circumferential displacements at Intertrochanteric region

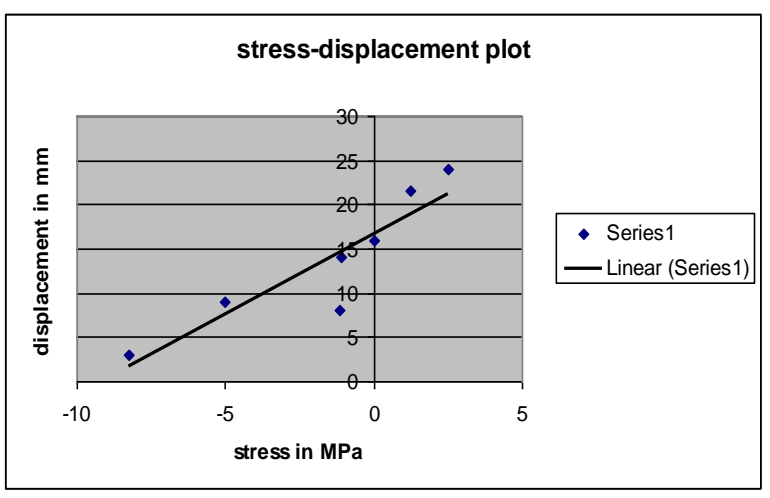

Fig-22: Stress-Displacement diagram at Intertrochanteric region

\section{CONCLUSIONS}

The work was based on the third generation standardized femur CAD model being provided by Rizzoli Orthopedic Institute. In order to improve the stress strain scenario in the proximal femur, a 3D analysis was carried out. Simulation of the Marten's study revealed that the highest stress formed in the absence of the cancellous tissue is almost double the value of stress formed with cancellous tissue. To approach a realistic model of the femur, CT scanned data was used for assigning material properties to the cancellous region. By locating salient geometric features on the CAD model with the VHP femur model, the material properties at four crucial locations were calculated and assigned to the current model. Thus the current model consisted of four regions of cancellous tissue with different material properties for the four sections.

A comparative study was made with the Lotz's model by taking into consideration two different sections near the head and neck of the femur. It was found that the highest stress value developed near the neck coincided with the value obtained using the current model when an adduction angle of 11 degrees was incorporated in the previous model discussed. Adduction angle is the angle made by the longitudinal axis of the femur shaft with the vertical during one legged stance. Since the present study concerned itself with fractures at the intertrochanteric region, the stresses and strains developed at the intertrochanteric region during one-legged stance was analyzed separately. It was found that the stresses and strains maintained a linear relationship throughout the outer periphery of a section obtained at the intertrochanteric region. Also, it was concluded that the intertrochanteric region is a vulnerable one owing to the stress accumulation present in the area.

It was established that the predominant type of stress in the femur is compressive rather than tensile. In all the cases discussed, it was found that the magnitude of stress developed was maximum at the anterior region of the femur, below the femoral head. On the other hand, the tensile stresses were predominant at the posterior region at the diaphyseal region and were of a lower magnitude, owing to the fact that it acted over a larger area. Also, it was found that orthotropic properties, when applied to the cortical tissue along with the adduction angle made the results approach the values of stresses found in the literature.

\section{REFERENCES:}

[1] Huiskes, R., Weinans, H., \& Van Rietbergen , B. (1992). The relationship between stress shielding and bone resorption around total hip stems and the effects of flexible materials, Stress Shielding and Bone Resorption, Vol.115, pp.534-542.

[2] Hampton S.J., Andriacchi T.P., Galante J.O. (1980) Three Dimensional Stress Analysis of the Femoral Stem of a Total Hip Prosthesis. Journal of Biomechanics, Vol. 13, pp.443-448. 
[3] Brown T., D., Hild G.L. (1983) Pre-Collapse Stress Redistributions in Femoral Head Osteonecrosis- A Three-Dimensional Finite Element Analysis. Journal of Biomechanical Engineering, Vol.105, pp.171-176.

[4] Brown T.D., Digioia A.M, (1984) A contact-coupled finite element analysis of the natural adult. Journal of Biomechanics, Vol.17, pp.437-448.

[5] McNamara, B.P., Cristofolini, L., Toni, A., \& Taylor, D. (1997). Relationship between bone-prosthesis bonding and load transfer in total hip reconstruction. Journal of Biomechanics, Vol.30, pp.621-630.

[6] Little R.B., Wevers H.W., Siu D., Cooke T.D.V (1986) A Three Dimensional Finite Element Analysis of the Upper Tibia, Journal of Biomechanical Eng. Vol.108, pp.111-119.

[7] Lotz J. C., Cheal, E.J.,\& Hayes W.C. (1991) Fracture prediction for the proximal femur using finite element models: Part 1- Linear analysis. Journal of Biomechanics, Vol.113, pp.353-360.

[8] Rohlmann A., Mossner U., Bergmann G., Kolbel R. (1982) Finite-element analysis and experimental investigation of stresses in a femur. Journal of Biomed, Vol.4, pp.241-246.

[9] Lotz J.C., (1988) Hip fracture risk predictions by X-ray Computed Tomography, Ph.D. Dissertation, Department of Mechanical Engineering, Massachusetts Institute of Technology.

[10] Keyak J.H., Skinner H.B. Three-dimensional finite element modeling of bone: effects of element size. Journal of Biomed. Eng, Vol.14, pp.483-489.

[11] Carter D.R., Hayes W.C (1977) The compressive behavior of bone as a two-phase porous structure. The journal of Bone and Joint structure.Vol.59-a, pp.954962.

[12] Ashman R.B., Cowin S.C., Van Buskirk W.C.,Rice J.C. (1984) A continuous wave technique for the measurement of the elastic properties of cortical bone. J. Biomechanics, Vol.17,pp.349-361.

[13] Burstein A.H., Reilly D.T., Martens M. (1976) Aging of bone tissue: mechanical properties. J Bone Joint Surg, Vol.59, pp82-86.

[14] Keyak J.H., Fourkas, M.G., Meagher J.M., Skinner, H.B.(1990) Validation of an Automated Method of Three-dimensional Finite Element Modeling of Bone. Journal of Biomedical Engineering, Vol.15, pp.505509.

[15] Reilly D.T. and Burstein A.H. (1975) The elastic and ultimate properties of compact bone tissue. J Biomechanics, pp.393-405.

[16] Ashman R.B., Corin J.D., Turner C.H. (1987) Elastic properties of cancellous bone: measurement by ultrasonic technique. J.Biomechanics, Vol.20, pp.979986.

[17] Martens M., Van Audekercke R., Delport P., De Meester P., Mulier J.C. (1983) The mechanical characteristics of cancellous bone at the upper femoral region. J. Biomechanics, Vol.16, pp 971-983.

[18] Hayes W.C., Snyder B., Levine B.M., Ramaswamy S. (1982) Stress morphology relationships in trabecular bone of the patella. John Willey, New York. Finite Elements in Biomechanics.

[19] Hayes W.C., Snyder B.,(1979) Correlations between stress and morphology in trabecular bone of the patella. Proceedings of the $25^{\text {th }}$ Annual Meeting of Orthopedic Research Society, ORS. Chicago, pp.88.

[20] Van Buskrik W.C., Ashman R.B. (1981) The elastic properties of bone. Mechanical properties of bone, Joint ASME-ASCE Bioengineering Conference, Boulder Colorado, American Society of Mechanical Engineers, New York.

[21] Rohl L., Larsen E., LInde F., Odgaard A., Jorgensen J. (1991) Tensile and compressive properties of cancellous bone. J. Biomechanics, Vol.24, pp 11431149.

[22] Brown T., and Ferguson A., (1980) Mechanical Property Distribution in the Cancellous Bone of the Human Proximal Femur. Orthop. Scand, Vol.51, pp.429-437.

[23] Rho J.Y., Ashman R.B., Turner C.H.(1991) Young's modulus of trabecular and cortical bone material: ultrasonic and microtensile measurements. J, Biomechanics. Vol.26, pp 155-168.

[24] Eric Wang, Three dimensional stress analysis of the proximal femur; MS Thesis, Buffalo University, USA.

\section{BIOGRAPHY:}

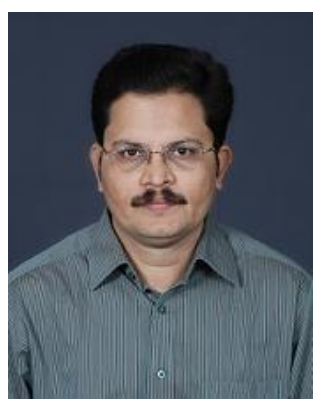

Sivakumar is an Associate Professor of Aerospace Engineering department in Amrita Vishwa Vidyapeetham university, Coimbatore, Tamil Nadu, India. $\mathrm{He}$ received his $\mathrm{Ph} . \mathrm{D}$ in Applied mechanics from Indian Institute of Technology madras, Chennai. He has around 19 years of experience in teaching and industry. He has published and presented papers in several referred international journals and conferences. He specializes in stress analysis, non-linear FE analysis and Composite materials and mechanics. He is a life member in 'The Institution of Engineers (India)' and Indian Society for Advancement of Materials and Processing Engineering (ISAMPE). 